\title{
Coder and Decoder of Block 3B4B with Auxiliary Channel
}

\author{
António D. Reis ${ }^{1}$, José F. Rocha², Atílio S. Gameiro², and Jose P. Carvallho \\ ${ }^{1}$ University of Beira Interior \\ ${ }^{2}$ University of Aveiro
}

\section{Abstract}

This work presents the coder and decoder of block 3B4B with auxiliary channel. The coder 3B4B converts an input 3 bits word in an output 4 bits word. It transmits an equal number of 1's and O's to provide a DC constant component. It increases the transitions number, improves the system quality and security. The objective is also to improve the system potentialities with an auxiliary channel to monitor the communication (alarm). The main channel is real, but the auxiliary channel is fictitious.

Keywords: Block codes, Digital systems, Transmission lines

Corresponding Author:

António D. Reis

adreis@ubi.pt

Received: 26 November 2019

Accepted: 13 May 2020

Published: 2 June 2020

Publishing services provided by Knowledge E

(c) António D. Reis et al. This article is distributed under the terms of the Creative Commons Attribution License, which permits unrestricted use and redistribution provided that the original author and source are credited.

Selection and Peer-review under the responsibility of the ICEUBI2019 Conference Committee.

\section{Introduction}

This work studies the coder and decoder of block 3B4B with auxiliary channel $[1,2,3,4$, $5,6,7,8,9,10]$. The auxiliary channel (CA) does not consume physical resources. It is only achieved by programming the appropriate $\mathrm{mBnB}$ code in the PROMs (programmable $\mathrm{ROM}$ ) of the encoder and decoder.

The code must satisfy the rule $n=m+1$ ( $m, n$ integers), to minimize the transmission elevation txo $=t x i{ }^{*} \mathrm{n} / \mathrm{m}$. Also, $\mathrm{n}$ must be even to guarantee a constant $\mathrm{DC}$ component with many transitions. Also, $m$ must be low to minimize the system complexity.

The coder converts a three bits word $3 \mathrm{~B}$ in a word 4B. Posteriorly, the decoder converts newly the four bits word $4 \mathrm{~B}$ in the original word $3 \mathrm{~B}$, recovering the initial sequence.

Fig.1 shows the communication system with relevance for the blocks coder and decoder 3B4B with auxiliary channel.

The coder improves certain characteristics such as:

- Constant DC component what avoids signal fluctuation.

S OPEN ACCESS

- Sufficient transitions for clock recovery and retiming. 


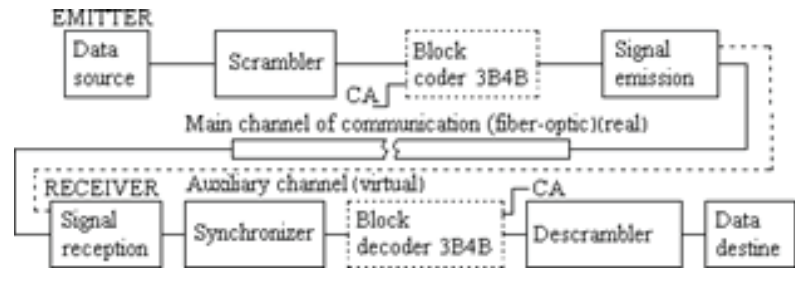

Figure 1: Communication system with the cod - dec 3B4B auxiliary

- Independent of the bits sequence.

- Decodification independent of the state.

- Multiplication of errors is low.

- Possibility of errors detection.

- Information for alignment of blocks / words.

In emitter, the data source provides the data, the scrambler becomes the spectrum input independent, the line coder equalizes the number of 1's and 0,s, the signal emission adapts the signal to the channel type.

In receiver, the signal receiver provides the electric signal, the synchronizer recoveries the clock, the decoder makes the inverse of the coder, the descrambler makes the inverse of the scrambler and the destination is the final.

The code 3B4B with auxiliary channel is the same normal code 3B4B, where only one word $3 \mathrm{~B}$ is coded differently, when the $\mathrm{CA}$ is zero $(C A=0)$ or one $(C A=1)$

We choose, for example, the word 3B number 7. Then, it is necessary that two forbidden words belong to the dictionary and codify the situation $C A=1$. We choose, for example, the words 1110, 0001 (Tab.1).

TABLE 1: Table of the code 3B4B with auxiliary channel

3B word
0- 000
1- 001
2- 010
3- 011
4- 100
5- 101
6- 110
7- 111
(7)- 111

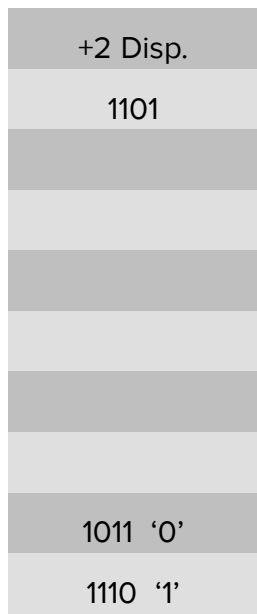

\begin{tabular}{|c|c|}
\hline & -2 Disp. \\
\hline & 0010 \\
\hline 0011 & \\
\hline 0101 & \\
\hline 0110 & \\
\hline 1001 & \\
\hline 1010 & \\
\hline 1100 & 0100 '0' \\
\hline & 0001 ' 1 ' \\
\hline
\end{tabular}


From a total of 16 words $4 B$ there are $10+2$ words for the dictionary $(+2 D=3, O D=6$, $-2 D=3$ ). There are 6-2 forbidden words that are [0000, (withdrawal 0001), 0111, 1000, (withdrawal 1110), 1111]. This table produces the followings tables of codification and decodification.

\section{Tables of Codification and Decodification}

With the code 3B4B auxiliary, we can obtain the table of codification 3B4B and the table of decodification 3B4B.

\subsection{Table of codification (PROM - Cod)}

A DC constant component implies to send an equal number of 1's and 0's. Then, after to send a positive disparity word, ignoring the 0 disparity word, is necessary to send a negative word and vice-versa (Tab.2).

This table is programmed in the PROM (Programmable Read Only Memory) of the coder.

\subsection{Table of decodification (PROM - Decod)}

In the decoder PROM must be programmed the corresponding table of decodification (Tab.3)

The words with don't care (11 - - -) are forbidden, can be associated with for example $(11---) \equiv(11010)$.

\section{Pair Coder and Decoder 3B4B}

To provide the operation of encoding and decoding tables is necessary to project (create) the hardware of the coder and decoder.

\subsection{Coder 3B4B}

The codification table is programmed in a PROM, having a flip flop that guards the information of the parity $\mathrm{P}$ (positive or negative) of the last transmitted word, according to the status diagram of Fig.2. 
TABLE 2: Table of codification 3B4B auxiliary (PROM-Cod)

\begin{tabular}{|c|c|c|c|c|c|}
\hline $\begin{array}{l}\mathrm{M}+3 \mathrm{~B} \\
\mathrm{CMA}_{2} \mathrm{~A}_{1} \mathrm{~A}_{0}\end{array}$ & \multicolumn{2}{|c|}{+2 Disp. $\mathrm{PB}_{3} \mathrm{~B}_{2} \mathrm{~B}_{1} \mathrm{~B}_{0}$} & $\begin{array}{c}0 \text { Disp. } \\
\mathrm{PB}_{3} \mathrm{~B}_{2} \mathrm{~B}_{1} \mathrm{~B}_{0}\end{array}$ & \multicolumn{2}{|c|}{-2 Disp. $\mathrm{PB}_{3} \mathrm{~B}_{2} \mathrm{~B}_{1} \mathrm{~B}_{0}$} \\
\hline $0-00000$ & 11101 & & & & \\
\hline $1-00001$ & & & 00011 & & \\
\hline $2-00010$ & & & 00101 & & \\
\hline 3- 00011 & & & 00110 & & \\
\hline 4- 00100 & & & 01001 & & \\
\hline 5- 00101 & & & 01010 & & \\
\hline 6- 00110 & & & 01100 & & \\
\hline 7- 00111 & 11011 & ‘ $0^{\prime}$ & & & \\
\hline 8- 01000 & & & & 00010 & \\
\hline 9- 01001 & & & 10011 & & \\
\hline 10- 01010 & & & 10101 & & \\
\hline 11- 01011 & & & 10110 & & \\
\hline $12-01100$ & & & 11001 & & \\
\hline 13- 01101 & & & 11010 & & \\
\hline 14- 01110 & & & 11100 & & \\
\hline 15- 01111 & & & & 00100 & ‘’’ \\
\hline 16- 10000 & 11101 & & & & \\
\hline $17-10001$ & & & 00011 & & \\
\hline $18-10010$ & & & 00101 & & \\
\hline 19- 10011 & & & 00110 & & \\
\hline 20- 10100 & & & 01001 & & \\
\hline 21- 10101 & & & 01010 & & \\
\hline 22- 10110 & & & 01100 & & \\
\hline 23- 10111 & 11110 & $‘ ’$ & & & \\
\hline 24- 11000 & & & & 00010 & \\
\hline 25- 11001 & & & 10011 & & \\
\hline 26- 11010 & & & 10101 & & \\
\hline 27- 11011 & & & 10110 & & \\
\hline 28- 11100 & & & 11001 & & \\
\hline 29- 11101 & & & 11010 & & \\
\hline 30- 11110 & & & 11100 & & \\
\hline 31- 11111 & & & & 00001 & ' 1 \\
\hline
\end{tabular}

This states diagram implemented in the flip flop controls the codification mode of the PROM (mode 0 or mode 1). 
TABLE 3: Table of decodification 3B4B auxiliary (Prom-Dec)

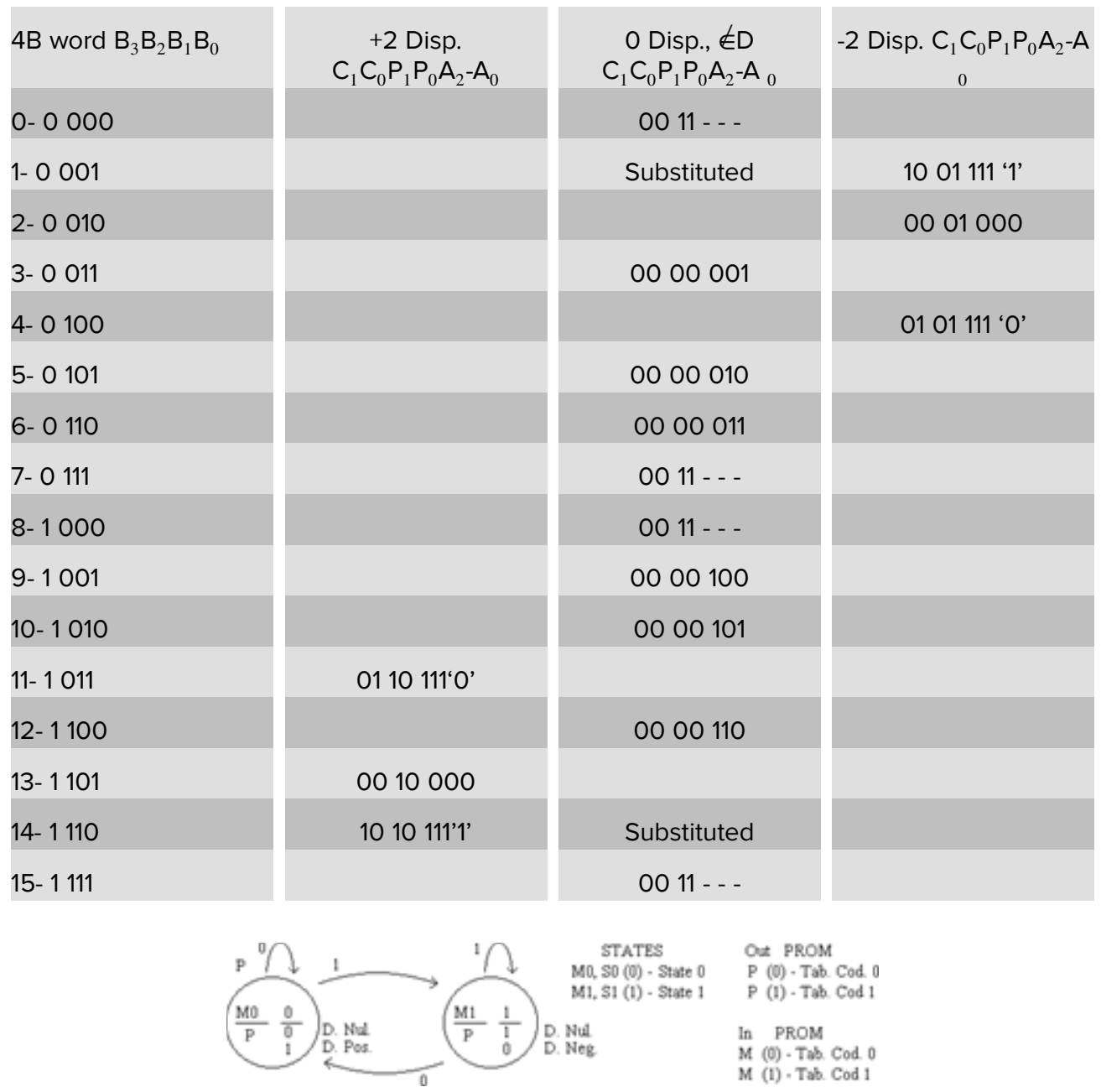

Figure 2: States diagram of the codification (memory element)

The PROM is the core of the coder. The table, in $M=0$ is the mode 0 and in $M=1$ is the mode 1. The table of codification is in the PROM and the states diagram (controller) is in the flip flop memory (Fig.3).

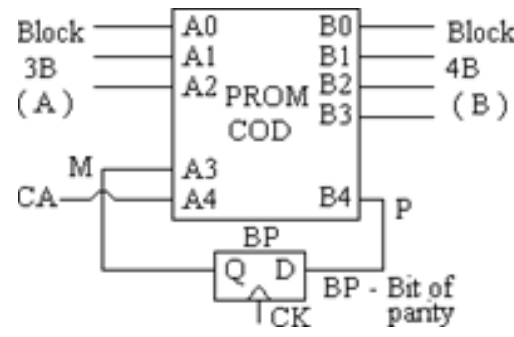

Figure 3: PROM with the codification table 3B4B aux and flip flop

The flip flop receives the disparity $\mathrm{P}$ of the anterior word and leads to the correct codification mode ( 0 or 1 ). If the disparity $P$ is null, it maintains the codification mode, otherwise it switches to the other codification mode. 
In the coder, the data enters in serial 3B, is converted to parallel, coded 3B4B, newly converted to serial $4 \mathrm{~B}$ and after sent. For this is necessary an input shift register serial - parallel, a normal register, memory PROM and an output parallel- serial shift register. So that this components (architecture) work correctly is necessary a controller based in a clock synthesizer involving two counters of module 3 and 4 and a PLL (Phase Lock Loop) (Fig.4).

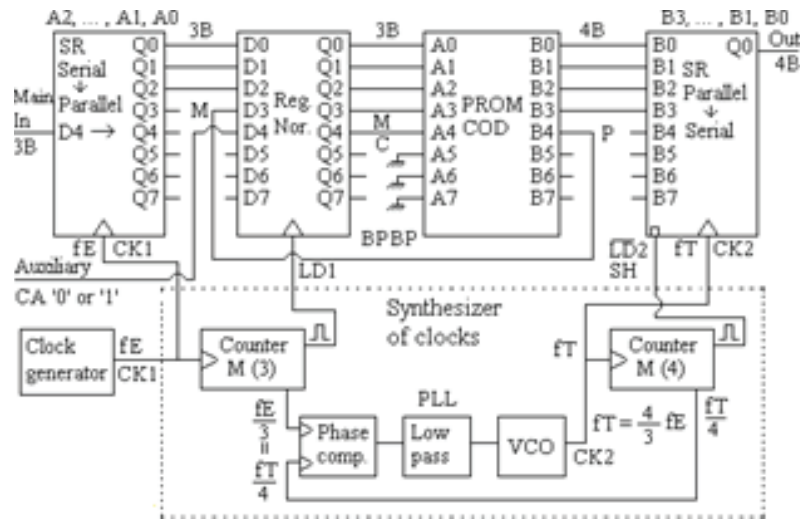

Figure 4: Coder 3B4B

The clock generator is the reference mark (beat) that marks the operation rhythm of the global system.

In the auxiliary channel $C A(7,15,23,31)$, when $C A=0$ are send the words of NORMAL (1011, 0100), when CA=1 are sent the words of ALARM (1110, 0001).

\subsection{Decoder 3B4B}

The decodification table 3B4B is programmed in a PROM (Decod), but is necessary that the input words are aligned (marked).

To align correctly the input words $4 \mathrm{~B}$ is necessary an error detector that detects the forbidden words that don't belong to the codification dictionary and still disrespect the accumulate disparity rule. When occur more than $x$ errors in $M$, there is suspicion of misalignment and then is activated the alignment mechanism.

The error detector receives information P1P0 of the words forbidden (not belongs to the dictionary) and still the disrespects to the accumulate disparity rule and leads to the error state Se, with output $Z=1$ (Fig.5).

The states diagram conduct to the states table, which leads to the circuit of errors detector (Fig.6) 


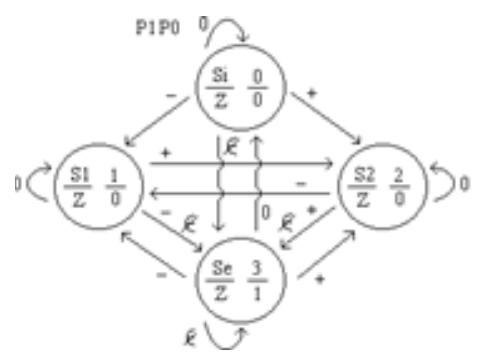

STATES

Si $(00)$ - Seate intid

S1 (01) - Sabe 1

S2 (10) - State 2

Se (11) - State enror

IN OUT

Pl P0 $(00)-0 \quad z(0)$ - Nerm

$P 1 P 0(01) \cdots z(1)$ - Erros

P1 P0 (10) *

P1 P0 (11) - E

Figure 5: States diagram of the errors detector

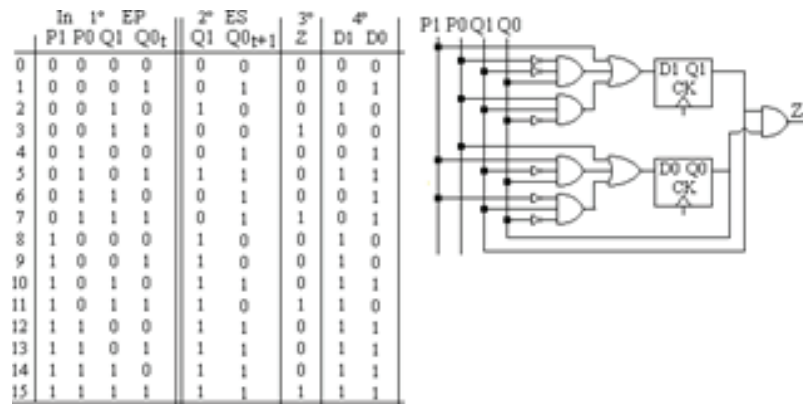

Figure 6: States table and respective errors detector

The errors detector was implemented with conventional logic (Karnaugh maps), but could be implemented in a PROM with 2 flip flops (states table) or in a PLD (states diagram).

The PROM (Decod) with the decodification table 3B4B and the errors detector has the configuration of Fig.7.

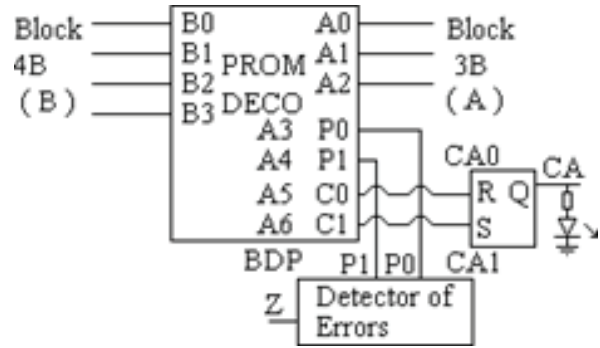

Figure 7: PROM with the decodification table 3B4B and Detector

So that the PROM (Decod) can work (function) correctly are needed various auxiliary circuits. In the decoder, the data enters in serial 4B, it is converted to parallel, decoded 3B4B and after converted newly to serial 3B recovering the original sequence.

For this, is need an input shift register serial - parallel, a normal register, a memory (PROM) and an output parallel - serial shift register. So that this components (architecture) work in perfect harmony is need the controller based in the clocks synthesizer of two counters of

The controller of module 4 can change provisionally the counting module for $4+1=5$, in order to make the word alignment, if there is more than $\mathrm{x}=8$ word errors in $\mathrm{M}=64$ words 


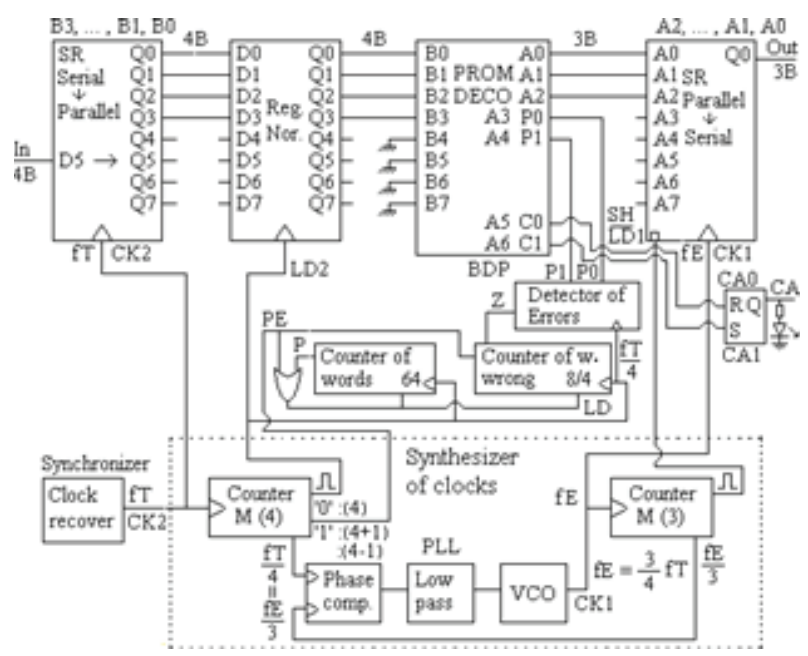

Figure 8: Decoder 3B4B

in the beginning and after 4 word errors in $M=64$. The clock recover (synchronizer) is the beat that marks the operation rhythm of the entire receiver.

In auxiliary channel CA, when appear the words of NORMAL (1011, 0100), $C_{1} C_{0}(S R)=01$, is made the latch Reset. When appear the words of ALARM (1110, 0001), $C_{1} C_{0}(S R)=10$ is made the latch Set (LED on). For other words $C_{1} C_{0}=00$ and the latch maintains the state.

\section{Project, Tests and Results}

We present bellow the project, tests and results [5].

\subsection{Project}

In the pair Coder - Decoder, such as in a digital communication system, is necessary to use good project techniques to create the hardware (architecture) with the able potentiality to execute the desired task. However, is still necessary to make them communicate using a clock synthesizer (controller) that gives unity to the system parts and whose clock is the beater that marks the rhythm.

After designed, the project was tested theoretically in the paper, with a sequence 1' and 0 alternated, however actually the simulation is the tool normally used.

Following, the pair Cod - Decod was mounted and tested in an ebonite breadboard. Finally, with previous performance guaranties, was implemented in printed circuit boards. 


\subsection{Tests}

The pair Cod - Decod was initially connected between itself by a cooper line. In the transitory state, during the power on, occur the inherent errors until to establish the word alignment, which after in the permanent state soon finished and no more appeared.

\subsection{Results}

The pair Cod-Decod was integrated in the global system with transmission by fibre optic with a gap (air space) that simulated $50 \mathrm{~km}$.

With a degraded signal in its relation signal - noise SNR in the minimum threshold of the CCITT (Comité Consultatif International Teleffonique Telégraphique) / ITU (International Telecomunication Union) the ratio error bits/ total bits BER (Bit Error Rate) of the system (BER - measured) was $10^{-15}$ and was lesser than the values allowed by the CCITT (BER - CCITT) of $10^{-12}$.

This errors 3 - 5 in a period of 24h, have nothing with the pair Cod - Decod, but yes with the fortuitous errors of the synchronizer decision when deciphers ' 1 ' or ' 0 ' in a signal strongly degraded and indecipherable at human eye.

\section{Conclusions}

We studied the block coder 3B4B with auxiliary channel.

The coder prepares the data to be transmitted with greater quality and security.

The pair coder decoder 3B4B is based in the code 3B4B programmed in a memory PROM and input serial-parallel and output parallel - serial.

The pair coder decoder needs in the emitter of a clock generator and in the decoder of a synchronizer (clock recovery) that are the reference clock of the synthesizer that controls the work rhythm of all the system.

In the transitory state of start, it arise the inherent alignments errors, that quickly disappear when the permanent state is reached.

With the pair Cod - Decod integrated in the global system, it arise some errors, which are related with the synchronizer decider that is disturbed by the noise.

Anyway, the obtained BER - measured was 10-15 what is lesser than the allowed BER - CCITT that is 10-12. 


\section{Acknowledgments}

The authors are grateful to the program FCT (Foundation for the sCience and Technology).

\section{References}

[1] D. A. Huffman, "A method for the construction of minimum redundancy codes", in Proceedings IRE Vol.40 pp.1098-1101, 1962.

[2] H. Lohscheller, "A subjectively adapted image communication system", IEEE Transactions on Comm. Com.32, pp.1316-1322, Dec. 1984

[3] S. A. Vanstone, P. Oorschot, "An introduction to error correcting codes with applications". Klumer Academic Publishers, 1989.

[4] ITU-T Recommendation H.261, "Video codec for audiovisual services at px64 kbps", 1993.

[5] A. Hefez, "Introdução à teoria dos códigos". UNICAMP, 1994.

[6] Joan L. Mitchell, William B. Pennebaker, Chad E. Fogg, Didier J. LeGall, “MPEG video compression standard", 1996.

[7] Roopali Garg, "Line Code for Optical Fiber Communication Systems”, IETE Journal of Education, Vol. 40, No. 3-4, PP. 93-100, Ponjab - India, Jul - Dec 1999.

[8] Demir Oner, "Criteria for Choosing Line Codes in Data Communication", Journal of Electrical and Electronics Engineering, Vol. 3, No. 2, PP. 843-857, Istambul 2003.

[9] V. Sneha Latha et al, "Performance Evaluation of Different Line Codes", Indian Journal of Computer Science and Engineering, Vol.2, No.4, PP. 575-588, India, Aug-Sep 2011.

[10] Ankit Gupta, Gurashish Singh, "Implementation and Analysis of Different Line Coding Schemes using Verilog", International Journal of Science, Engineering and Technology Research, Vol. 5, I. 2, PP. 395-401, India, Feb. 2016. 